\title{
Lesiones quísticas sincrónicas en los maxilares: reporte de casos
}

Synchronous cystic lesions in the jaws: case reports

Lesões císticas sincrônicas nos maxilares: relato de casos

Marcos Martins CURI ${ }^{1}$

Daniel Henrique KOGA ${ }^{2}$

Anthony Froes Benites CONDEZO 3

Beethoven Estevão COSTA ${ }^{3}$

Heitor Albergoni da SILVEIRA ${ }^{4}$

Camila Lopes CARDOSO ${ }^{5}$

${ }^{1}$ Cirujano Bucal y Maxilofacial en el Hospital Santa Catarina de São Paulo, Brasil

${ }^{2}$ Cirujano Bucal y Maxilofacial en el Hospital Santa Catarina de São Paulo, Brasil; Alumno de Doctorado en el área de Cirugía y Traumatologia Bucomaxilofacial, Centro Universitário UNISAGRADO,17011-160 Bauru - SP, Brasil

${ }^{3}$ Alumno de Doctorado en el área de Cirugía y Traumatologia Bucomaxilofacial, Centro Universitário UNISAGRADO, 17011-160 Bauru - SP, Brasil

${ }^{4}$ Alumno de Maestría en el área de Diagnóstico y Cirurgía de la Universidade Estadual Paulista, Araraquara - SP, Brasil

${ }^{5}$ Profesora en el Departamento de Cirugía y Traumatologia Bucomaxilofacial, Centro Universitário UNISAGRADO, 17011-160 Bauru - SP, Brasil

\section{Resumen}

La asociación de lesiones odontogenéticas afectando los huesos maxilares en un mismo paciente, es raro. El objetivo de este trabajo fue relatar dos casos de lesiones sincrónicas, afectando a un mismo paciente. En el primer caso, el paciente presentó un queratoquiste odontogénico en la mandíbula y un quiste del conducto nasopalatino, y en el segundo caso, el paciente presentó un ameloblastoma en la mandíbula y un quiste del conducto nasopalatino. Ambos casos fueron tratados a través de la remoción total de las lesiones, microscopicamente revelaron los respectivos diagnósticos y ahora estan siendo controlados, con la ausencia de recidivas después de cuatro años.

Descriptores: Queratoquiste Odontogénico; Ameloblastoma; Quiste del Conducto Nasopalatino.

\section{Resumo}

A associação de lesões odontogenéticas afetando os ossos maxilares em um mesmo paciente é rara. O objetivo deste trabalho foi relatar dois casos de lesões sincrônicas, acometendo o mesmo paciente. No primeiro caso, o paciente apresentava ceratocisto odontogênico na mandíbula e cisto do ducto nasopalatino e, no segundo caso, o paciente apresentava ameloblastoma na mandíbula e cisto do ducto nasopalatino. Ambos os casos foram tratados com a remoção das lesões, revelaram microscopicamente os respectivos diagnósticos e agora estão sendo acompanhados, não havendo recidivas após quatro anos.

Descritores: Ceratocisto Odontogênico; Ameloblastoma. Cisto do Ducto Nasopalatino.

\section{Abstract}

The association of odontogenetic lesions affecting the maxillary bones in the same patient is rare. The aim of this paper is to report two cases of synchronous lesions affecting the same patient. In the first case, the patient presented an odontogenic keratocyst in the jaw and a nasopalatine duct cyst, and in the second case, the patient presented an ameloblastoma in the jaw and nasopalatine duct cyst. Both cases were treated through the total removal of the lesions, microscopically revealed the respective diagnoses and now they are being controlled, with absence of recurrence after four years.

Descriptors: Odontogenic Keratocyst; Ameloblastoma; Nasopalatine Duct Cyst.

INTRODUCIÓN

Los quistes del desarrollo, son lesiones relativamente comunes que afectan a los huesos maxilares. En cuanto a su epidemiología, el queratoquiste odontogénico (QO) y el quiste del conducto nasopalatino (QCN), son quistes que se encuentran con frecuencia ${ }^{1}$, siendo el origen de los mismos diferente. El quiste del conducto nasopalatino o quiste del canal incisivo, es la lesión quística no odontogénica más común en los maxilares, descrita inicialmente por Meyer en 1914². Es una lesión originada de los restos embrionarios del conducto nasopalatino y se ubica en la región anterior del maxilar como una lesión radiolúcida, unilocular, bien delimitada, con bordes radiopacos, pudiendo presentarse en forma de corazón debido a la superposición de la espina nasal anterior ${ }^{3,4}$.

El QO fue descrito por primera vez en $1956^{5,6}$, clasificado como un quiste odontogénico. Frente a su comportamiento recidivante en el año 2005, la OMS (Organización Mundial de la Salud) lo clasificó como tumor odontogénico queratoquístico ${ }^{7}$. Sin embargo, después de 12 años de observación y estudios sobre esa lesión, los autores concluyeron recientemente que por su comportamiento no merece ser considerado de agresivo y por lo tanto, volvio a su clasificación inicial ${ }^{8}$. Considerando su aspecto radiográfico, se presenta como una lesión radiolúcida bien definida, con márgenes festoneados, multilocular o unilocular. Una peculiaridad de esta lesión, es su crecimiento intramedular en sentido antero-posterior, por tal motivo, no causa expansión de las corticales óseas en la mayoría de los casos ${ }^{9}$.

El ameloblastoma, otra lesión odontogénica osteolítica, es considerado el segundo tumor odontogénico más común y presenta un comportamiento recidivante $y$ agresivo localmente. Las lesiones mencionadas presentan etiologías diferentes y no totalmente claras. Se cree que un factor genético pueda participar de la etiología de estas lesiones.

La asociación de lesiones
odontogenéticas afectando los huesos
maxilares, en un mismo paciente, es rara, por lo tanto, el objetivo de este trabajo fue relatar dos casos de lesiones sincrónicas afectando a un mismo paciente. 


\section{REPORTE DE CASO}

\section{- Caso 1}

Paciente leucoderma de sexo masculino, 33 años de edad, fue conducida para evaluación de una lesión ubicada en el cuerpo de la mandíbula del lado derecho, que fue diagnosticada por el examen radiográfico panorámico. En el examen físico intrabucal, en la región retromolar derecha se observaron, mucosas normales y ausencia de expansión de las corticales óseas. La radiografía panorámica reveló una lesión radiolúcida, unilocular bien definida, en la región del cuerpo de la mandíbula del lado derecho, midiendo aproximadamente $4.5 \times 2.5 \mathrm{~cm}$, extendiéndose desde la raíz del primer molar (46) hasta la corona del tercer molar no erupcionado (48) con desplazamiento del canal mandibular (Figura 1). La misma radiografía mostró otra imagen radiolúcida unilocular entre las raíces de los incisivos centrales superiores (Figura 1).

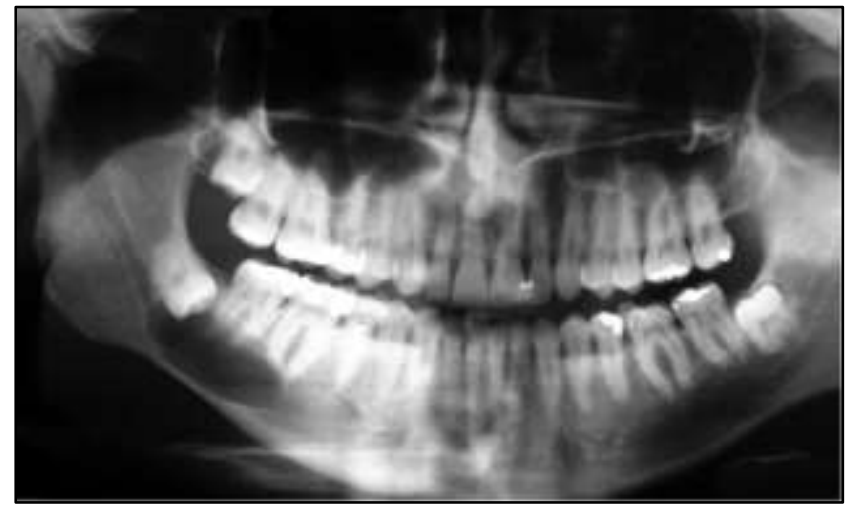

Figura 1: Radiografía panorámica que muestra lesiones en la mandíbula y en la región anterior del hueso maxilar.

La tomografía computarizada, reveló detalladamente las lesiones hipodensas bien delimitadas, en la región del cuerpo de la mandíbula del lado derecho y en la región anterior del hueso maxilar (Figura 2 y 3 ).

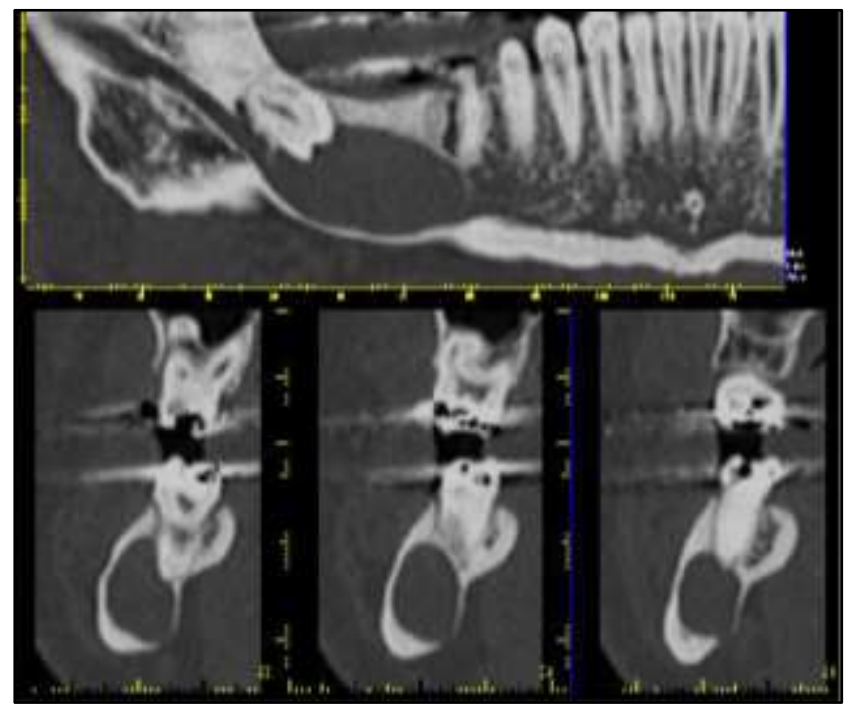

Figura 2: Tomografía computarizada demostrando una lesión cística hipodensa con límites bien definidos.

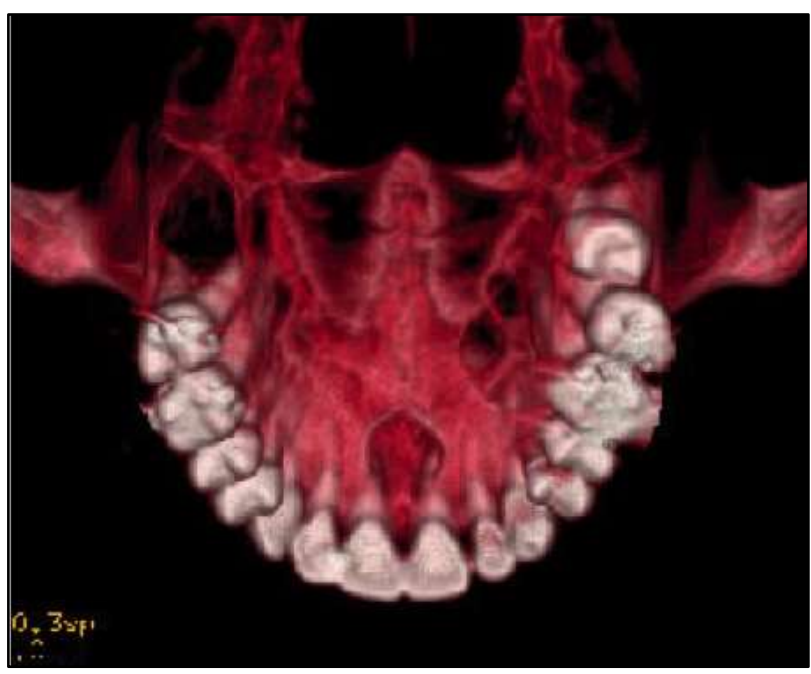

Figura 3: Reconstrucción 3D ilustrando la lesión en la región anterior del hueso maxilar.

Las hipótesis diagnósticas fueron de quiste dentígero y QO para la lesión en la mandíbula y de QCN para la lesión en el maxilar. Fue realizado biopsia incisional en ambas lesiones. La lesión de la mandíbula demostró microscópicamente una fina capa de epitelio estratificado escamoso con queratina en la parte basal, con un aspecto ligeramente empalizado y cápsula fibrosa conteniendo muchos vasos y quistes satélites. La lesión del maxilar, reveló una lesión quística con cápsula fibrosa revestida por una capa de epitelio estratificado escamoso. Las zonas de hemorragia, algunas células inflamatorias y los haces nerviosos se encontraron en la pared quística. El diagnóstico fue de $\mathrm{QO}$ y $\mathrm{QCN}$, respectivamente. El tratamiento consistió en la enucleación de ambas lesiones. El paciente está siendo controlado y no hubo evidencia de recidiva después de 18 meses de tratamiento.

\section{- Caso 2}

Paciente de género masculino, 18 años de edad, fue atendido quejándose de dolor en la región posterior de la mandíbula izquierda, relatando dos meses de evolución. En el examen clínico presentó dolor a la palpación y un discreto aumento de volumen en la región retromolar. El examen radiográfico evidenció una lesión radiolúcida multiloculada, con aspecto de panales de abeja, de aproximadamente $8 \times 4 \mathrm{~cm}$, límites irregulares, extendiéndose de la región del diente 4.6 hasta la apófisis coronoides, englobando también el canal mandibular (Figura 4). Una segunda lesión radiolúcida fue observada durante el examen radiográfico, localizada en la región anterior del maxilar, midiendo aproximadamente $3 \times 3 \mathrm{~cm}$ de diámetro, bien delimitada con aparente envolvimiento de las raíces de los incisivos superiores. Clínicamente no fue posible observar ninguna alteración, ni 
sintomatología. Las hipótesis diagnósticas de la alteración en la mandíbula fueron: ameloblastoma, mixoma o QO. En el maxilar, el diagnóstico presuntivo fue QCN.

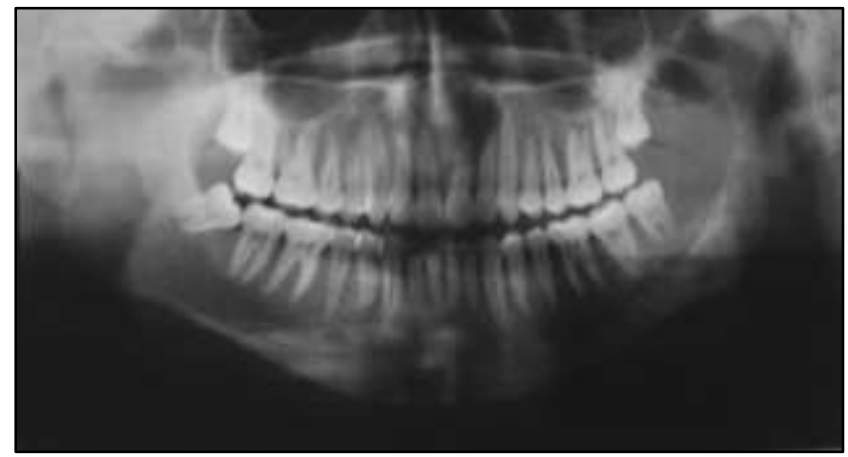

Figura 4. Radiografía panorámica con lesiones en el lado izquierdo de la mandíbula y en la región anterior del hueso maxilar.

El paciente fue sometido a biopsia de la lesión mandibular y los cortes microscópicos revelaron una proliferación de células epiteliales similares a ameloblastos, formando cordones anastomosados, confirmando la hipótesis de ameloblastoma, del tipo histológico plexiforme. Después de dos meses del diagnóstico, se realizó la cirugía del paciente bajo anestesia general, realizando su resección marginal. En el mismo tiempo quirúrgico, se realizó la enucleación de la lesión quística que se ubicaba en el maxilar. El análisis de los especímenes confirmó ameloblastoma del tipo plexiforme en la mandíbula y QCN en el maxilar. El paciente ya tiene dos años de control y hasta el momento no presentó recidivas de las lesiones.

DISCUSSION

La teoría más aceptada para el desarrollo de los quistes y tumores odontogénicos, se relaciona al desarrollo de la lámina dental y sus restos, originando islas epiteliales comúnmente encontradas en la encía y el ligamento periodontal, por eso, no es descrito exactamente la interconexión entre el origen de tal patología con tales restos epiteliales ${ }^{2,4,10}$. Anatómicamente es encontrado en la región del ramo y ángulo mandibular, el QO corresponde al $11 \%$ de todos los quistes encontrados en la mandíbula, presentando una alta tasa de recidiva ${ }^{6,11,12}$, radiográficamente es encontrado como una lesión radiolúcida uni o multilocular con los bordes definidos ${ }^{13}$. La región más comprometida por el ameloblastoma también es la región posterior de la mandíbula, que tiene un crecimiento lento, siendo asintomático, y generalmente es encontrado en exámenes radiográficos odontológicos de rutina ${ }^{14}$.

La patogénesis del QCN viene siendo discutida con teorías divergentes en cuanto al origen epitelial de esta lesión y cuáles son los factores asociados. Hay un consenso actual de que se origina del epitelio de restos de un órgano primitivo del olfato, encontrado en los animales inferiores (órgano vomer-nasal de Jacobson), en forma de conductos revestidos por epitelio, cordones epiteliales, restos epiteliales 0 combinaciones ${ }^{1}$. Traumatismo local, presencia de infección en la región, factores raciales y genéticos, fueron todos sugeridos como causas posibles. Sin embargo, la teoría de la proliferación espontánea de restos epiteliales parece ser la explicación más probable para el desarrollo de la lesión ${ }^{15}$. Esta teoría viene contraponer la teoría fissural, aceptada por mucho tiempo. La teoría fissural se fundamenta en el encarcelamiento de epitelio durante el desarrollo embriológico de la cara, durante la unión de los procesos embriológicos; en estos lugares, restos epiteliales podrían haber quedado aislados y por factores desconocidos pudieron proliferar dando origen a un quiste. Actualmente, se sabe que, durante el desarrollo embriológico de la cara, ocurre la nivelación de los procesos embriológicos con brote de proliferación del mesenquima, excluyendo así la posibilidad de aprisionamiento del epitelio, descartando la existencia de esos quistes "fissurales".

Considerando el pronóstico de las lesiones relatadas en este trabajo, existe una gran diferencia durante el comportamiento clínico. Mientras que el QO y el ameloblastoma presentan alto índice de recidiva, el QCN es una lesión que difícilmente resulta en recidivas, si es enucleado completamente. En el presente caso, ninguna de las lesiones presentaron recidiva, vale resaltar que el $\mathrm{QO}$, se trato con una terapia adyuvante, a través de la crioterapia, frente a su comportamiento recidivante descrito en la literatura.

Aunque el $\mathrm{QO}$ y el QCN presentan etiología y comportamiento biológicos diferentes, en el presente caso ambas lesiones quísticas aparecieron de forma sincrónica en los maxilares. Los exámenes de imagen y anatomopatológicos, son importantes para un adecuado diagnóstico y tratamiento. Resaltamos que el QO y ameloblastoma, ante las altas tasas de recidiva que presentan en la literatura, se hace necesario un control a largo plazo del paciente.

El tratamiento más eficaz del QO es la enucleación, con o sin terapia adyuvante ${ }^{16}$, siendo también la conducta ideal para el $\mathrm{QCN}^{2}$. Por la alta tasa de recidiva presentada en la literatura, el tratamiento del ameloblastoma debe ser cuidadosamente planificado, pudiendo ocurrir de forma conservadora o radical ${ }^{17,18}$, 
teniendo como forma de tratamiento, desde la enucleación, enucleación con aplicación de solución de Carnoy $^{19}$, resección marginal e incluso la mandibulectomía.

La asociación de las lesiones descritas indica la existencia de un componente genético, relacionado a las lesiones de desarrollo. Raramente, se han reportado asociados en la literatura, por lo que más estudios deben realizarse así como investigaciones genéticas en los pacientes afectados. Los casos reportados continúan siendo controlados, sin presencia de recidiva o desarrollo de nuevas lesiones.

\section{REFERENCIAS}

1. Shear M. Cistos da Região Bucomaxilofacial Diagnóstico e Tratamento. 3.ed. São Paulo: Santos, 1999.

2. Shylaja S, Balaji K, Krishna A. Nasopalatine duct cyst: report of a case with review of literature. Indian J Otolaryngol Head Neck Surg. 2013;65(4):385-8.

3. Martins MD, Russo MP, Bussadori SK, Fernandes KPS, Missawa GTM, Martins MAT. Nasopalatine duct cyst: report of case and literature review. Rev Inst Ciênc Saúde. 2007;25(2):193-7.

4. Suter VG, Sendi P, Reichart PA, Bornstein MM. The nasopalatine duct cyst: an analysis of the relation between clinical symptoms, cyst dimensions, and involvement of neighboring anatomical structures using cone beam computed tomography. J Oral Maxillofac Surg. $2011 ; 69(10): 2595-603$

5. Philipsen HP. OM keratocystedr (Kolesteratomer) e kaeberne. Tandlaegebladet. 1956;60:963-71.

6. Pindborg J, Hansen J. Studies on odontogenic cyst epithelium. II. Clinical and roentgenographic aspects of odontogenic keratocysts. Acta Pathol Microbiol Scand. 1963; 58:283.

7. Barnes L, Eveson JW, Reichart P, Sidransky D. World Health Organization Classification of Head and Neck Tumours. Pathology and Genetics of Head and Neck Tumours. 3rd ed. Lyon: IARC Press; 2005. p 160-1, 206-7.

8. Wright JM, Vered M. Update from the 4th Edition of the World Health Organization Classification of Head and Neck Tumours: Odontogenic and Maxillofacial Bone Tumors. Head Neck Pathol. 2017;11(1):68-77.

9. Menon S. Keratocystic Odontogenic Tumours: Etiology, Pathogenesis and Treatment Revisited. J Maxillofac Oral Surg. 2015;14(3):541-7.

10. Stoelinga PJW. The treatment of odontogenic keratocysts by excision of the overlying, attached mucosa, enucleation, and treatment of the bony defect with Carnoy solution. J Oral Maxillofac Surg. 2005;63:1662-6.

11. Carlson ER, Oreadi D, McCoy JM. Nevoid Basal Cell Carcinoma Syndrome and the Keratocystic Odontogenic Tumor. J Oral Maxillofac Surg. 2015;73(12Suppl):S77-86.

12. Maurette PE, Jorge $J$, de Moraes $M$. Conservative treatment protocol of odontogenic keratocyst: a preliminary study. J Oral Maxillofac Surg. 2006;64(3):379-83.

13. Chrcanovic BR, Gomez RS. Recurrence probability for keratocystic odontogenic tumors: An analysis of 6427 cases. J Craniomaxillofac Surg. 2017;45(2):244-51.

14. Kim J, Nam E, Yoon S. Conservative management (marsupialization) of unicystic ameloblastoma: literature review and a case report Maxillofac Plast Reconstr Surg. 2017; 39(1):38

15. Mesquita JA, Luna AHB, Nonaka CFW, Godoy GP, Alves PM. Aspectos clínico, tomográfico e histopatológicos do cisto do ducto nasopalatino. Braz j otorhinolaryngol. 2014;80(5):448-50.

16. Kinard BE, Chuang SK, August M, Dodson TB. For treatment of odontogenic keratocysts, is enucleation, when compared to decompression, a less complex management protocol? J Oral Maxillofac Surg. 2015;73(4):641-8.

17. Lau SL, Samman N. Recurrence related to treatment modalities of unicystic ameloblastoma: a systematic review. Int J Oral Maxillofac Surg. 2006;35:681-90.

18. Zhang J, Gu Z, Jiang L, Zhao J, Tian M, Zhou J et al. Ameloblastoma in children and adolescents. $\mathrm{Br} J$ Oral Maxillofac Surg. 2010;48(7):549-54.

19. de Paulo LFB, Oliveira MTF, Rodrigues ÁR, Zanetta-Barbosa D. Treatment of an extensive unicystic ameloblastoma in a 7-year-old child: the best approach? $\mathrm{Br} \mathrm{J}$ Oral Maxillofac Surg. 2015;53(3):292-4.

\section{CONFLICTO DE INTERÉS}

Los autores declaran no tener conflictos de interés.

AUTOR DE CORRESPONDENCIA

\section{Camila Lopes Cardoso}

Centro Universitário Sagrado Coração - Unisagrado

Calle Irmã Arminda 10-50,

17011-160 Bauru, São Paulo, Brasil

E-mail: cardoso_lopes@yahoo.com.br 